\title{
¿Mejora la comunicación con las redes sociales?
}

Does communication improve with the use of social networks?

A comunicação melhora com as redes sociais?

EDITORIAL

\section{Cecilia Ugalde}

Universidad del Azuay (Ecuador)

cugalde@uazuay.edu.ec

orcid.org/0000-0001-7716-6023

\section{Resumen:}

Las redes sociales vistas desde distintos espacios nos indican que más allá de los problemas que se pueden presentar por su mal uso, son más los beneficios que pueden traer a distintos espacios de comunicación, para lo que se deben tener en cuenta ciertos procesos y buenas prácticas. Es preciso que aprendamos a utilizar las redes sociales para beneficio de entornos sociales, comerciales, públicos y privados.

\section{PALABRAS CLAVE}

Comunicación, Redes sociales.

\section{Abstract:}

The social networks seen from different spaces indicate that, beyond the problems that can arise due to their misuse, there are more benefits that they can bring to different communication spaces, for which certain processes and good practices must be taken into account. We must learn to use social networks for the benefit of social, commercial, public and private environments.

\section{KEYWORDS}

Communication, Social networks, Social Media.

\section{Resumo}

As redes sociais vistas a partir de diferentes espaços indicam que para além dos problemas que podem surgir devido ao seu uso indevido, existem mais benefícios que podem trazer para os diferentes espaços de comunicação, para os quais determinados processos e boas práticas devem ser tidos em consideração. Precisamos aprender a usar as redes sociais para beneficiar os ambientes sociais, comerciais, públicos e privados.

\section{PALABRAS-CHAVE}

Comunicação, Redes sociais 
Las redes sociales son parte del día a día de millones de personas en todo el mundo (We are social, 2020), y su uso se ha estudiado no solamente a nivel interpersonal (Candrasari, 2020), o comunitario (Chan et al., 2020; Haşiloğlu et al., 2020), sino también a nivel empresarial, donde se ha encontrado que tienen un impacto positivo en las relaciones dentro de las empresas, así como en el rendimiento de sus empleados (Chen \& Wei, 2020) y la relación con sus clientes, stakeholders y comunidad (Guo et al., 2020; Hila et al., 2020). Por otra parte, también se han estudiado las consecuencias del abuso en el uso de redes sociales (de Pedro \& Martín, 2018), así como prácticas poco éticas (Eckert, 2017; Oksanen et al., 2020), o la infodemia, caracterizada por la abundancia de información sobre un tema concreto, como por ejemplo alrededor del COVID-19 y su asociación con problemas de salud mental como depresión o ansiedad (Gao et al., 2020) entre otras cosas.

Con todo esto salta a la luz la necesidad de integrar buenas prácticas y regulaciones que permitan utilizar de mejor manera y optimizar los beneficios que las redes sociales pueden tener en las distintas áreas de la sociedad, más allá de la comunicación en espacios informales, hasta espacios comerciales y públicos (Dwivedi et al., 2020; Lovari \& Bowen, 2020; Lovari \& Valentini, 2020).

El número 20 de Obra Digital aborda distintos espacios en los que se demuestra que las redes sociales pueden, en efecto, contribuir a una mejor comunicación, e incluso a solucionar problemas ocasionados por el mal uso de las mismas redes sociales, como podemos ver en el artículo sobre los efectos de la desinformación sobre la imagen de marca y reacción de tres empresas del sector alimentario ante una crisis de comunicación. También se destaca la importancia a nivel empresarial del uso de redes sociales más informativas en detrimento de las más conversacionales en un estudio de la estructura de las redes sociales en las compañías del IBEX 35.

Por otro lado, se analizan distintas estrategias promocionales en Instagram en un escenario de competencia en dos continentes; y queda en evidencia que muchas empresas chilenas no fomentan una verdadera comunicación con sus interlocutores en Facebook, sino que más bien se limitan a una comunicación unidireccional que no genera engagement.

En otros entornos vemos que las redes sociales abren nuevos espacios de integración y de conservación de tradiciones entre mujeres kurdas no escolarizadas; mientras que también se analiza el uso que los estudiantes universitarios dan a las redes sociales durante períodos de movilidad académica.

Encontramos además un artículo sobre las percepciones sociales de la selección chilena de fútbol como marca que posee un alto grado simbólico capaz de unir a una sociedad fragmentada como la chilena, y, finalmente, presentamos un estudio sobre la comunicación interna como área estratégica para la innovación a través de la gestión del cambio y la felicidad organizacional.

De esta manera, Obra Digital 20, correspondiente a los meses de febrero - agosto 2021, presenta ocho artículos enviados desde tres países (Chile, España, Turquía) con representación de tres continentes. Se publica en sus versiones originales en español e inglés, además de una versión completa en inglés. Esperamos que sea de su interés. 


\section{Referencias}

Candrasari, Y. (2020, March). Mediated Interpersonal Communication: A New Way of Social Interaction in the Digital Age. In 2nd International Media Conference 2019 (IMC 2019) (pp. 537-548). Atlantis Press. https://doi. org/10.2991/assehr.k.200325.041

Chan, A. K., Nickson, C. P., Rudolph, J. W., Lee, A., \& Joynt, G. M. (2020). Social media for rapid knowledge dissemination: early experience from the COVID-19 pandemic. Anaesthesia, 75(12), 1579-1582. https://doi. org/10.1111/anae.15057

Chen, X., \& Wei, S. (2020). The impact of social media use for communication and social exchange relationship on employee performance. Journal of Knowledge Management, 24(6), 1289-1314. https://doi.org/10.1108/ JKM-04-2019-0167

de Pedro, A. I. I., \& Martín, T. M. (2018). Redes sociales y aplicaciones de móvil: uso, abuso y adicción. Revista INFAD de Psicología. International Journal of Developmental and Educational Psychology., 4(1), 203-212. https://doi. org/10.17060/ijodaep.2018.n1.v4.1295

Dwivedi, Y. K., Ismagilova, E., Hughes, D. L., Carlson, J., Filieri, R., Jacobson, J., ... \& Wang, Y. (2020). Setting the future of digital and social media marketing research: Perspectives and research propositions. International Journal of Information Management, 102168. (In Press) https://doi. org/10.1016/j.jijinfomgt.2020.102168

Eckert, S. (2017). Fighting for recognition: Online abuse of women bloggers in Germany, Switzerland, the United Kingdom, and the United States. New Media \& Society, 20(4), 1282-1302. https://doi. org/10.1177/1461444816688457

Gao, J., Zheng, P., Jia, Y., Chen, H., Mao, Y., Chen, S., Wang, Y., Fu, H., \& Dai, J. (2020). Mental health problems and social media exposure during COVID-19 outbreak. Plos one, 15(4), e0231924. https://doi.org/10.1371/ journal.pone.0231924

Guo, Y., Fan, D., \& Zhang, X. (2020), Social media-based customer service and firm reputation. International Journal of Operations \& Production Management, 40(5), 575-601. https://doi.org/10.1108/IJOPM-04-2019-0315

Haşiloğlu, M. A., Çalhan, H. S., \& Ustaoğlu, M. E. (2020). Determining the Views of the Secondary School Science Teachers about the Use of Social Media in Education. Journal of Science Education and Technology, 29(3), 346354. https://doi.org/10.1007/s10956-020-09820-0 
Hia, E. P. D., Prestiadi, D., Maghfiroh, S. I., \& Oktaviani, V. M. (2020, November). The Use of Information and Communication Technology Through Website and Social Media as Public Relations Information Media. In 2nd Early Childhood and Primary Childhood Education (ECPE 2020) (pp. 269-274). Atlantis Press.

Lovari, A., \& Bowen, S. A. (2020). Social media in disaster communication: A case study of strategies, barriers, and ethical implications. Journal of Public Affairs, 20(1), e1967. https://doi.org/10.1002/pa.1967

Lovari, A., \& Valentini, C. (2020). Public sector communication and social media: Opportunities and limits of current policies, activities, and practices. The Handbook of Public Sector Communication, 315-328. https://doi. org/10.1002/9781119263203.ch21

Oksanen, A., Oksa, R., Savela, N., Kaakinen, M., \& Ellonen, N. (2020). Cyberbullying victimization at work: Social media identity bubble approach. Computers in human behavior, 109, 106363. https://doi.org/10.1016/j. chb.2020.106363

We are social. (2020). Digital 2020: 3.8 billion people use social media. https:// wearesocial.com/blog/2020/01/digital-2020-3-8-billion-people-usesocial-media 